\title{
A NONLINEAR VISCOELASTIC MODEL AND NON-EQUILIBRIUM ENTROPIES
}

\author{
Mengran Sun ${ }^{\mathrm{a}, \mathrm{b}}$, David Jou ${ }^{\mathrm{b}}$, Jinjun Zhang, ${ }^{\mathrm{a}, *}$ \\ ${ }^{a}$ National Engineering Laboratory for Pipeline Safety/ MOE Key Laboratory of Petroleum \\ Engineering/Beijing Key Laboratory of Urban Oil and Gas Distribution Technology, China \\ University of Petroleum-Beijing, 102249 Beijing, China \\ ${ }^{b}$ Departament de Fisica, Universitat Autonoma de Barcelona, 08193 Bellaterra, Catalonia, \\ Spain
}

\begin{abstract}
We show that the thermodynamic conjugate of the extra stress is a more suitable independent variable than the extra stress itself for the thermodynamic description of some nonlinear models of viscoelasticity. This brings more precision to the knowledge of non-equilibrium thermodynamic potentials, and provides an illustration of a recently proposed conservation-dissipation formalism which allows bringing extended irreversible thermodynamics to the nonlinear regime.
\end{abstract} Keywords: Entropy; Nonlinear viscoelasticity; Extended irreversible thermodynamics; Conservation-dissipation formalism

2010 MSC: 00-01, 99-00

\section{Introduction}

The suitable selection of independent variables is one of the basic problems in non-equilibrium thermodynamics [1. Rheological systems are especially challenging from this point of view, because they have a very rich and dynamic 5 microscopic structure. For a microscopic understanding and simulation of their behavior, structural variables such as the conformation tensor $\underline{\underline{C}}$, proportional to $\langle\vec{R} \vec{R}\rangle$, with $\vec{R}$ the end-to-end vector of the macromolecules and $\langle\cdots\rangle$ standing for

\footnotetext{
${ }^{*}$ Corresponding author

Email address: zhangjj@cup.edu.cn (Jinjun Zhang)
}

Preprint submitted to J. Non-Newtonian Fluid Mech.

January 13, 2016

(C) 2016. This manuscript version is made available under the Elsevier user license http://www.elsevier.com/open-access/userlicense/1.0/ 
the average over all molecules [2, 3, 4, 5], are especially suitable and useful. The conformation tensor $\underline{\underline{C}}$ is related to the stress tensor $\underline{\underline{\sigma}}$ in a simple way (usually, through a linear algebraic relation). The use of this variable, and other related variables describing further structural details of the system, in combination with molecular dynamics simulations of the flowing system and with especially wide and powerful macroscopic thermodynamic formalisms as GENERIC [2, 3, 4, 5], has been at the basis of systematic analyses of very complex rheological systems involving different time scales(see, for instance, 6, 7, 8, 9, 10, 11, 12]).

More traditional non-equilibrium and macroscopically focused thermodynamic approaches to rheological modelling [12, 13, 14, are less specific and detailed than the mentioned recent approaches. They focus on more macroscopic variables, mainly the stress tensor - or the several contributions to it -, and are mainly interested in the compatibility of rheological models with the second law of thermodynamics $[12,13,14,15,16$, without the explicit knowledge of the microstructural variables. Whereas more microscopically-minded approaches aim to detailed descriptions of different physical systems, more macroscopicallyminded theories aim to search for the features which are common to a wide diversity of systems, despite having less predictive power in the particular details.

A topic of particular interest in the macroscopic theories is the contribution of the non-equilibrium variables to the entropy and the entropy flux. Through their contribution to the entropy, these non-equilibrium variables contribute also to the equations of state and may modify the phase diagram of flowing rheological fluids. For instance, polymer solubility or miscibility is known to be influenced by the imposed shear rate or shear stress [13, 15].

A phenomenological alternative to the explicit use of internal structural variables, which are not always easy to identify, measure, or control, is to directly 35 use the extra stress, or some related macroscopic non-equilibrium quantities, as an additional independent thermodynamic variable, leading to non-equilibrium expressions for entropy 12, 13, 14. In particular, in extended thermodynamics, $\underline{\underline{\sigma}}$ is used as additional variable in the entropy $s$, which is of the form $s(u, \underline{\underline{\sigma}})$. 
When $\underline{\underline{\sigma}}$ and $\underline{\underline{C}}$ are linearly related, one may go from the use of $\underline{\underline{\sigma}}$ to the use of

$40 \underline{\underline{C}}$ by means of a Legendre transform [17]. The aim of this paper is to go to the non-linear regime in the framework of such macroscopic theories and explore the most suitable independent variables. In section 2 , we briefly review the relation between linear Maxwell viscoelastic equation and the non-equilibrium entropy of extended thermodynamics. In section 3, we present the nonlinear version of (1)

45 considered here, and illustrate how the entropy (2) fails to describe it. In section 4 , by using its corresponding thermodynamic conjugate instead of $\sigma$ itself, we present a different version of non-equilibrium entropy which shows in a direct way the compatibility of the nonlinear viscoelastic equation with the second law of thermodynamics. In Section 5 we make some concluding comments on the new version of entropy, Legendre transforms, and GENERIC thermodynamic formalism for nonlinear constitutive equations.

\section{Linear viscoelasticity and extended entropy}

Here, in particular, we focus our attention on the form of macroscopic nonequilibrium entropy in connection with viscoelasticity. Though the form of entropy plays a central role in the analysis of compatibility of constitutive equations with the second law, its explicit form is rarely studied in detail, as the dynamical aspects of rheological equations, being directly observable and with relevant physical consequences, focus the attention of most researches.

The simplest model for viscoelasticity is the Maxwell model, which is char60 acterized by the well-known equation [5, 12, 13, 2.

$$
\tau \frac{\mathbf{D} \underline{\underline{\underline{\sigma}}}}{\mathbf{D} t}+\underline{\underline{\sigma}}=2 \eta \underline{\underline{D}}
$$

in which $\underline{\underline{\sigma}}$ is the extra stress tensor, $\tau$ is the viscoelastic relaxation time, $\eta$ is the shear viscosity, $\underline{\underline{D}}$ is the rate of deformation tensor, which represents the symmetric part of the velocity gradient $\nabla \vec{v}$ as $\underline{\underline{D}}=\frac{1}{2}(\nabla \vec{v}+\vec{v} \nabla)$, and whose second invariant is the so-called shear rate $\dot{\gamma}$, and $\mathbf{D} \underline{\underline{\sigma}} / \mathbf{D} t$ stands for frameinvariant forms of the time derivative defined as $\mathbf{D} \underline{\underline{\sigma}} / \mathbf{D} t \equiv \mathbf{d} \underline{\underline{\sigma}} / \mathbf{d} t-a[(\nabla \vec{v})$. 
$\left.\underline{\underline{\sigma}}+\underline{\underline{\sigma}} \cdot(\nabla \vec{v})^{\mathrm{T}}\right]$, with $\mathrm{d} / \mathrm{d} t$ the material time derivative. In [18, 19, 20, 21], the upper-convected time $(a=1)$ was used for the viscoelastic models to be frameinvariant, but other time derivatives like the co-rotational one, or the lower convected one $(a=-1)$ could also be used [2, [3, 4, 15, 12, 13, 14]. Since the macromolecular systems usually have several relaxational degrees of freedom contributing to $\underline{\underline{\sigma}}$, model $[1]$ is generalized by taking $\underline{\underline{\sigma}}=\sum \underline{\underline{\sigma}}_{i}$, with each contribution $\underline{\underline{\sigma}}_{i}$ being described by an equation like (1), with its own $\eta_{i}$ and $\tau_{i}$. The several relaxation times may span a wide range of time scales. Here, for the sake of simplicity, and because the corresponding extension is straightforward, we will consider a single degree of freedom.

In the linear situation that $\eta$ and $\tau$ are independent of time and deformation, for compressible fluid, the corresponding Gibbs equation for the non-equilibrium thermodynamic entropy $s$ per unit mass of the fluid in extended irreversible thermodynamics (EIT) is [13, 22, 23, 24]

$$
\mathrm{d} s(u, \underline{\underline{\sigma}})=T^{-1} \mathrm{~d} u-\frac{\tau}{2 \eta T \rho} \underline{\underline{\sigma}}: \mathrm{d} \underline{\underline{\sigma}}
$$

80 of the fluid, the thermodynamic description based on $u$ and $\underline{\underline{\sigma}}$ (or on $T$ and $\underline{\underline{\sigma}}$ ) may be more useful from a macroscopic point of view, because it is a directly measurable variable. 


\section{Non-linear viscoelastic equation}

In nonlinear situations, $\eta$ and $\tau$ may depend on fluid microstructure which, in its turn, depends on the shear condition. Then, $\eta$ and $\tau$ may be taken to depend on some characteristic microstructure variables or, alternatively, on the shear rate $\dot{\gamma}$ as in shear-thinning fluids, or on $\gamma$ and $\dot{\gamma}$ as in gel-sol transitions. An open thermodynamic question is which will be the extension of the Gibbs equation (2) for nonlinear extension, i.e. which is the form of entropy more directly related to nonlinear versions of equation (1).

For nonlinear viscoelasticity, we will consider a nonlinear extension of (1) as proposed by Marrucci et al. [18, 19, 20, 21, and we will ask for the corresponding extension of the entropy (2), related to a recent conservation-dissipation formalism (CDF) of irreversible thermodynamics [25], and compare it to extended irreversible thermodynamics (EIT) [13, 22, 23, 24] as well as the GENERIC formalism [5, 3, 4].

The simplest nonlinear extension of (1) would be to use (1) itself, but with $\eta$ and $\tau$ changing with time and deformation, or depending on the microstructure. However, from an experimental point of view, this model is not satisfactory enough. Instead of (1), Marrucci et al. [18, 19, 20, 21] have proposed for systems with structure-dependent relaxation times, to use the following generalized version

$$
\eta \frac{\mathbf{D}}{\mathbf{D} t}\left(\frac{\tau}{\eta} \underline{\underline{\sigma}}\right)+\underline{\underline{\sigma}}=2 \eta \underline{\underline{D}}
$$

which becomes (1) when $\eta$ and $\tau$ are constant (in particular, they take for $\mathrm{D} / \mathrm{D} t$ the upper-convected or contra-variant time derivative). The ratio $\eta / \tau$ is the so-called elastic modulus $G$, and its reciprocal is the steady-state compliance $J$. Equation (3) is justified in the framework of network theories, as applied to polymer melts and concentrated solutions, where viscosity and relaxation times strongly depend on the microstructure, thus leading to a shear rate dependence $\eta(\dot{\gamma})$ and $\tau(\dot{\gamma})$. In [18, 19, 20, 21, microscopic description of the system was proposed with emphasis on the dynamics, i. e. on the rheological aspects, and compared to experiments showing its practical suitability. Our aim, instead, 
will be focused on the most suitable form of non-equilibrium entropy being compatible with equation (3). Additional nonlinear effects would also be added to (3), as for instance, terms of the form $\underline{\underline{\sigma}} \cdot \underline{\underline{\sigma}}$, but the non-equilibrium entropy is known to be related to the relaxational term of the constitutive equation [13, 14, 22], so that we focus our attention on this term.

Note that the suitable thermodynamic potential related to (3) is not the entropy (2). Indeed, if the Gibbs equation (2) is used as a starting point for the thermodynamic analysis, one gets for the evolution equation of $\underline{\underline{\sigma}}$, equation (1) with $\eta(\dot{\gamma})$ and $\tau(\dot{\gamma})$ instead of equation (3). To see this, recall that the evolution equation for $u$ is the energy balance equation [5, 12, 13, 2, 22, 23, 26 .

$$
\rho \dot{u}=-\nabla \cdot \vec{q}+\underline{\underline{\sigma}}: \underline{\underline{D}}
$$

where $\vec{q}$ is the heat flux, and the last term describes viscous dissipation per unit volume and time. The evolution equation for $s$ according to $(2)$ is

$$
\rho \dot{s}=T^{-1} \rho \dot{u}-\frac{\tau}{2 \eta T} \underline{\underline{\sigma}}: \underline{\underline{\sigma}}
$$

In fact, in order that the several terms in (5) are frame-invariant, the $\underline{\underline{\dot{\sigma}}}$ in $(5)$ should be interpreted as a frame-invariant time derivative as mentioned below (1) because $\underline{\underline{\sigma}}$ is a second-order tensor (in contrast, the several time derivatives reduce to the material time derivative for scalar quantities $s$ and $u$.)

140 Introducing (4) into (5) one finds

$$
\rho \dot{s}=\vec{q} \cdot \nabla T^{-1}+T^{-1} \underline{\underline{\sigma}}: \underline{\underline{D}}-\frac{\tau}{2 \eta T} \underline{\underline{\sigma}}: \underline{\underline{\dot{\delta}}}-\nabla \cdot\left(T^{-1} \vec{q}\right)
$$

The first and last terms on the right-hand side come from the term $-T^{-1} \nabla \cdot \vec{q}$ obtained when (4) is introduced into the first term of the right-hand side of (5).

If one compares (6) to the general evolution equation for the entropy, namely [5, 12, 13, 22, 23, 26],

$$
\rho \dot{s}+\nabla \cdot \overrightarrow{J^{s}}=\sigma^{s}
$$

with $\vec{J}^{s}$ entropy flux and $\sigma^{s}$ entropy production per unit volume and time, one finds $\vec{J}^{s}=\vec{q} / T$, which is the usual expression for the entropy flux, and

$$
\sigma^{s}=\vec{q} \cdot \nabla T^{-1}+T^{-1} \underline{\underline{\sigma}}:\left(\underline{\underline{D}}-\frac{\tau}{2 \eta} \underline{\underline{\underline{\sigma}}}\right) \geq 0
$$


In (8), we impose $\sigma^{s} \geq 0$, which is the usual requirement of the second law of thermodynamics [5, 12, 13, 22, 23, 26].

The simplest way of satisfying requirement (8) is to assume constitutive equations for the flux $\vec{q}$ and the extra stress tensor $\underline{\underline{\sigma}}$ in terms of their respective conjugate forces, i.e. the terms multiplying them in the bilinear expression (8), according to the usual procedure of non-equilibrium thermodynamics [5, 13, 26]. One gets in this way

$$
\vec{q}=L_{1} \nabla T^{-1}
$$

for the vectorial part related to $\vec{q}$, and

$$
T^{-1} \underline{\underline{\sigma}}=L_{2}\left(\underline{\underline{D}}-\frac{\tau}{2 \eta} \underline{\underline{\sigma}}\right)
$$

some modification is necessary to analyze the thermodynamic consistency of the nonlinear expression (3). In particular, we will explore a change in the form of entropy, as proposed by Zhu et al. [25, which is aimed to the description of nonlinear situations.

\section{Conservation-dissipation formalism and nonlinear viscoelasticity}

Zhu et al. 25. have proposed the conservation-dissipation formalism (CDF), which reduces to Extended Thermodynamics in the linear case but which differs from it in some crucial aspects in the nonlinear domain. We will show here that this formalism provides indeed a suitable framework for the nonlinear viscoelastic equation (3), and we will pay special attention to the corresponding form of non-equilibrium entropy, as compared to the extended entropy (2). 
In CDF model the non-equilibrium entropy is not dependent on $u$ and $\underline{\underline{\sigma}}$, as in EIT, but on $u$ and $\underline{\underline{w}}$, with $\underline{\underline{w}}$ being the thermodynamic conjugate of $\underline{\underline{\sigma}}$, which is discussed below.

\subsection{Entropy representation}

Here, we will separately consider two options for $\underline{\underline{w}}$, namely, the conjugate in entropy representation and the conjugate in internal energy representation. To find the relation between $\underline{\underline{w}}$ and $\underline{\underline{\sigma}}$, let us rewrite 2 as

$$
\mathrm{d} s=T^{-1} \mathrm{~d} u+\underline{\underline{w}}: \underline{\underline{\sigma}}
$$

Here, $\underline{\underline{w}}$ is the thermodynamic conjugate of $\underline{\underline{\sigma}}$ with respect to $s$, defined by

$$
\underline{\underline{w}}=\left(\frac{\partial s}{\partial \underline{\underline{\sigma}}}\right)
$$

By comparing 11$]$ with 2 one sees that $\underline{\underline{w}}$ is related to $\underline{\underline{\sigma}}$ as

$$
\underline{\underline{w}}=-\frac{\tau}{2 \eta T \rho} \underline{\underline{\sigma}}
$$

In the steady state, where $\underline{\underline{\sigma}}=2 \eta \underline{\underline{D}}, \underline{\underline{w}}$ may be identified as $\underline{\underline{w}}=-(\tau / \rho) \underline{\underline{D}}$. Using $\underline{\underline{w}}$ instead of $\underline{\underline{\sigma}}$ as a variable, Zhu et al. 25] write a generalized entropy $\tilde{s}(u, \underline{\underline{w}})$, whose corresponding differential form (Gibbs equation) is

$$
\mathrm{d} \tilde{s}=T^{-1} \mathrm{~d} u+\underline{\underline{s}}_{w}: \mathrm{d} \underline{\underline{w}}
$$

where $\underline{\underline{s}}_{w} \equiv(\partial s / \partial \underline{\underline{w}})$. If one combines the time derivative of 14 with the energy balance equation (4) one obtains

$$
\rho \dot{\tilde{s}}=\vec{q} \cdot \nabla T^{-1}+T^{-1} \underline{\underline{\sigma}}: \underline{\underline{D}}+\rho \underline{\underline{s}} w \underline{\underline{\underline{\dot{w}}}}-\nabla \cdot\left(T^{-1} \vec{q}\right)
$$

Here, as in (5), $\underline{\underline{\dot{w}}}$ stands not for the material time derivative, but for the frameinvariant derivative $\mathrm{D} \underline{\underline{w}} / \mathrm{D} t$, but we keep $\underline{\underline{\underline{w}}}$ for notational simplicity. By following the method leading from (6) to 8 ), we obtain in this case for the entropy production

$$
\sigma^{\tilde{s}}=\vec{q} \cdot \nabla T^{-1}+T^{-1} \underline{\underline{\sigma}}:\left[\underline{\underline{D}}+\rho_{\underline{\underline{s}}}: \underline{\underline{\dot{w}}}\right] \geq 0
$$



functions, as

$$
\tilde{s}_{w}(s, \underline{\underline{w}})=\alpha_{1}^{\prime} \underline{\underline{U}}+\alpha_{2}^{\prime} \underline{\underline{w}}+\alpha_{3}^{\prime} \underline{\underline{w}} \cdot \underline{\underline{w}}
$$

where the scalar coefficients $\alpha_{i}$ may be functions of $u, I_{\underline{\underline{w}}}$ and $I I_{\underline{\underline{w}}}$, being $I_{\underline{\underline{w}}}=$ $\operatorname{tr}(\underline{\underline{w}}), I I_{\underline{\underline{w}}}=\operatorname{tr}(\underline{\underline{w}} \cdot \underline{\underline{w}})$ the scalar invariants of the tensor $\underline{\underline{w}}$. However, to be tensorial part of 18 to

$$
T^{-1} \underline{\underline{\sigma}}=\tilde{L}_{2}(\underline{\underline{D}}+\rho \alpha \underline{\underline{\underline{w}}})
$$

with $\tilde{L}_{2}$ positive. By taking $\alpha=T, \tilde{L}_{2}=2 \eta / T$ and using 13 , we get

$$
\underline{\underline{\sigma}}=\left[2 \eta \underline{\underline{D}}-T \frac{\mathrm{D}}{\mathrm{D} t}\left(\frac{\tau \underline{\underline{\underline{\sigma}}}}{\eta T}\right)\right]
$$

which may be rewritten as

$$
\underline{\underline{\sigma}}+T \eta \frac{\mathrm{D}}{\mathrm{D} t}\left(\frac{\tau \underline{\underline{\sigma}}}{\eta T}\right)=2 \eta \underline{\underline{D}}
$$

When $T$ is constant, 21 reduces to (3). The situation of $T$ changing with time is also of interest, especially in systems with sol-gel transitions due to the dissolved particles which may precipitate and aggregate at low enough temperatures, in such a way that their physical properties may strongly depend on temperature [27, 28].

\subsection{Internal energy representation}

An ambiguity in the definition of the thermodynamic conjugate of $\underline{\underline{\sigma}}$ arises if instead of using the entropy representation, as in Section 3.1, one uses the 
energy representation. In this case, (2) could be rewritten as

$$
\mathrm{d} u=T \mathrm{~d} s+\underline{\underline{w}}^{\prime}: \mathrm{d} \underline{\underline{\sigma}}
$$

with

$$
\underline{\underline{w}}^{\prime}=\left(\frac{\tau}{\eta \rho}\right) \underline{\underline{\sigma}}
$$

being the conjugate of $\underline{\underline{\sigma}}$ in this representation. If one uses $\underline{\underline{w}}^{\prime}$ instead of $\underline{\underline{w}}$ defined in $(13)$, as an independent variable of entropy $s^{*}\left(u, \underline{\underline{w}}^{\prime}\right)$, a thermodynamic analysis analogous to that of Section 3.1 would be

$$
\mathrm{d} s^{*}=T^{-1} \mathrm{~d} u-\underline{\underline{s}}_{w^{\prime}}: \mathrm{d} \underline{\underline{w}}^{\prime}
$$

where $\rho \underline{\underline{s}}_{w^{\prime}}=\alpha^{\prime} \underline{\underline{\sigma}} / T$. This leads to an entropy production

$$
\sigma^{s^{*}}=q \cdot \nabla T^{-1}+T^{-1} \underline{\underline{\sigma}}:\left(\underline{\underline{D}}+\rho \alpha^{\prime} \underline{\underline{\underline{w}}}^{\prime}\right) \geq 0
$$

and the corresponding stress equation

$$
T^{-1} \underline{\underline{\sigma}}=L_{2}^{\prime}\left(\underline{\underline{D}}+\rho \alpha^{\prime} \underline{\underline{\underline{w}}}\right)
$$

with $L_{2}^{\prime}$ positive. For $\alpha^{\prime}=-1$ and $L_{2}^{\prime}=\eta / T$, equation (3) is obtained.

Thus the entropy $\tilde{s}(u, \underline{\underline{w}})$ leads to $(21)$ and the entropy $s^{*}\left(u, \underline{\underline{w}}^{\prime}\right)$ leads to (3). For constant $T$ both equations coincide, but they differ when $T$ changes with time. A comparison of the predictions of $(3)$ and of (11) with experimental observations in situations with time-dependent temperature would clarify whether $\underline{\underline{w}}$ in (12) or $\underline{\underline{w}}^{\prime}$ in $(23)$ is more useful as independent variable of the entropy.

\section{Concluding remarks}

As final remarks related to the non-equilibrium entropies, we comment on Legendre transforms, and on GENERIC formalism in connection with the analysis about non-equilibrium entropy, in order to get a wider perspective on it. 


\subsection{Legendre transforms}

230 set a relation between equivalent thermodynamic formalisms in terms of different variables, namely, by replacing a variable (like $u$ ) by its thermodynamic conjugate $\left(T^{-1}\right.$ in the entropy representation), or replacing $s$ by $T$, in the energy representation. Since in 14 the thermodynamic conjugate of $\sigma$ is used, it related by means of a Legendre transform.

The main difference between EIT and CDF is that the former one uses $\underline{\underline{\sigma}}$ as independent variable, whereas the second one uses its thermodynamic conjugate $\underline{\underline{w}}$. Both theories coincide in the linear case. Now, we focus our attention on the

are not thermodynamically equivalent, except in the case where $\eta / \tau$ does not depend on the structure or on the shear rate. In contrast, a Legendre-transform 
relation between the internal-variable entropy $s(u, \underline{\underline{C}})$, having as variable the

conformation tensor $\underline{\underline{C}}$ of a dilute polymer solution, and the EIT entropy $s(u, \underline{\underline{\sigma}})$ was shown in [30, in the linear regime.

\subsection{GENERIC formalism}

GENERIC formalism is a powerful and elegant tool for the analysis of highly nonlinear situations, expressing the evolution of any variable $A_{i}$ as

$$
\frac{d A_{i}}{d t}=\{A, E\}+[A, S]
$$

with $E$ the energy, $S$ the entropy, $\{\cdots\}$ the Poisson (antisymmetric) brackets and $[\cdots]$ a dissipative (symmetric) brackets. Given a set of dynamical variables $A_{i}$ and the form of $E$ and $S, 30$ sets a number of physical basic requirements on the evolution equations. Nonlinearities in GENERIC are more diverse than in $(3)$, because nonlinear terms, for instance, like $\underline{\underline{\sigma}} \cdot \underline{\underline{\sigma}}$ may be also present. Here, 265 we consider the particular situation where the nonlinear viscoelastic equation (3) is used. Several years ago, in the framework of the GENERIC formalism [5] 3. 4], it was already pointed out by Ottinger and Grmela [4] that the use of the extra stress $\underline{\underline{\sigma}}$ as independent variable of the non-equilibrium entropy in EIT was not satisfactory beyond the linear regime. In fact, they found equation 270 (3) for dilute polymer solution as a particular result of the GENERIC approach. For that particular case, they proposed to use as independent variable $\underline{\underline{\sigma}} / n k_{\mathrm{B}} T$, $n$ being the macromolecular number density (per unit volume of solution) and $k_{\mathrm{B}}$ Boltzmann constant, instead of $\underline{\underline{\sigma}}$. Since for polymer dilute solutions one has $\eta=n k_{\mathrm{B}} T \tau$, this suggestion was leading to the use of $\tau \underline{\underline{\sigma}} / \eta$ as independent thermodynamic variable. In the conservation-dissipation formalism it seems $\tau \underline{\underline{\sigma}} / \eta T$ that (i. e. $\underline{\underline{w}}$ ) would be a more suited variable than $\underline{\underline{\sigma}}$ itself. Both proposals lead to result (3) in the isothermal situation. In fact, in the nonlinear domain, GENERIC is wider and more systematic than EIT or CDF, but much more demanding and richer in some aspects especially because the Hamiltonian requirement on the evolution equations leads to a deep structure in the nondissipative terms (which is lacking in usual thermodynamic theories) and other 
nonlinearities than those included in (3) may arise [5, 3, 4, 31].

In particular, Grmela and Ottinger 4] obtain the non-equilibrium entropy for the polymer contribution

$$
s_{\mathrm{p}}=\frac{1}{2} n k_{\mathrm{B}} \int\left[\operatorname{tr}\left(\frac{\underline{\underline{\Pi}}}{n k_{\mathrm{B}} T}\right)+\ln \operatorname{det}\left(\underline{\underline{U}}-\frac{\underline{\underline{\underline{I}}}}{n k_{\mathrm{B}} T}\right)\right] \mathrm{d}^{3} \vec{r}
$$

with $\underline{\underline{U}}$ the unit tensor, and $\underline{\underline{\Pi}}$ the polymer pressure tensor, whose non-equilibrium part is $\underline{\underline{\sigma}}$, for which they find

$$
\underline{\underline{\Pi}}=n k_{\mathrm{B}} T(C \underline{\underline{C}}) \cdot(\underline{\underline{U}}-C \underline{\underline{C}})
$$

where $\underline{\underline{C}}$ is the macromolecular conformation tensor, and $C$ a scalar quantity properly chosen so that at equilibrium $C \underline{\underline{C}}=\underline{\underline{U}}$. Note that $(32)$ is more general then the usual microscopic expression [5, 13]

$$
\underline{\underline{\Pi}}=n k_{\mathrm{B}} T(\underline{\underline{U}}-C \underline{\underline{C}})
$$

290 $\underline{\underline{\Pi}}$ or $\underline{\underline{\sigma}}$ may be especially useful from a practical macroscopic perspective when its expression in terms of internal variables related to the microstructure of the particular fluid is not directly controllable or not explicitly known. 


\section{Acknowledgements}

Mengran Sun and Jinjun Zhang acknowledge the financial support of National Science Foundation of China (Grant 51304224, 51534007 and 51134006). David Jou acknowledges the financial support of the Spanish Ministry of Economy and Competitiveness under grant FIS2012-32099. Together we also acknowledge the financial support from China Scholarship Council.

\section{References}

[1] R. Luzzi, A. Vasconcello, J. Casas-Vázques, D. Jou, On the selection of the state space in non-equilibrium themodynamics, Physica A 298 (1988) 111-137. doi:10.1016/S0378-4371(97)00530-X.

[2] A. N. Beris, S. J. Edwards, Thermodynamics of flowing fluids with internal microstructure, Oxford University Press, New York, 1994.

[3] M. Grmela, H. C. Ottinger, Dynamics and thermodynamics of complex fluids. i. development of a general formalism, Phys. Rev. E 56 (1997) 66206632. doi:10.1103/PhysRevE.56.6620.

[4] H. C. Ottinger, M. Grmela, Dynamics and thermodynamics of complex fluids. ii. illustrations of a general formalism, Phys. Rev. E 56 (1997) 66336655. doi:10.1103/PhysRevE.56.6633.

[5] H. C. Ottinger, Beyond equilibrium thermodynamics, John Wiley, Hoboken, 2005.

${ }_{325}$ [6] V. G. Mavrantzas, D. N. Theodorou, Atomistic simulation of polymer melt elasticity: calculation of the free energy of an oriented polymer melt, Macromolecules 12 (1998) 6310-6332. doi:10.1021/ma9714878.

[7] J. A. Kulkarni, A. N. Beris, New approach for simulating chain conformation in dense polymers using fully populated lattice models, Comput. Phys. 12 (1998) 641-651. doi:10.1063/1.168747. 
[8] V. G. Mavrantzas, H. C. Ottinger, Atomistic monte carlo simulations of polymer melt elasticity: their non-equilibrium thermodynamics generic formulation in a generalized cononical ensemble, Macromolecules 35 (2002) 960-975. doi:10.1021/ma010688f.

[9] P. Ilg, H. C. Ottinger, M. Kroger, Systematic time-scale-bridging molecular dynamics applied to flowing polymer melts, Phys. Rev. E 79 (2009) 1-8. doi:10.1103/PhysRevE.79.011802

[10] P. Ilg, M. Kroger, Molecularly derived constitutive equation for lowmolecular polymer melts from thermodynamically guided simulations, J. Rheology 55 (2011) 69-93. doi:10.1122/1.3523485.

[11] N. Germann, L. Cook, A. N. Beris, Nonequilibrium thermodynamic modeling of the strcture and rheology of concentrated wormlike micellar solutions, J. Non-Newtonian Fluid Mech. 196 (2013) 51-57. doi:10.1016/j.jnnfm. 2012.12 .010

[12] J. Verhás, Thermodynamics and rheology, Kluwer, Dordrecht, 1998.

[13] D. Jou, J. Casas-Vázquez, M. Criado-Sancho, Thermodynamics of fluids under flow, 4th Edition, Springer, Berlin, 2011.

[14] B. C. Eu, Generalized Thermodynamics: the thermodynamics of irreversible processes and generalized hydrodynamics, Kluwer, Dordrecht, 2002.

[15] A. Onuki, Phase transitions of fluids in shear flow, J. Phys.: Condens. Matter 9 (1997) 6119-6157. doi:10.1088/0953-8984/9/29/001.

[16] O. Manero, J. Pérez-Lopez, J. Escalante, J. Puig, F. Bautista, A thermodynamic approach to rheology of complex fluids: The generalized bmp model,

355 \. J. Non-Newtonian Fluid Mech. 146 (2007) 22-29. doi:10.1016/j.jnnfm. 2007.02 .012 
[17] J. Casas-Vázquez, L. F. del Castillo, D. Jou, M. Criado-Sancho, Legendre transforms in the thermodynamics of flowing polymer solutions, Phys. Rev. E 63 (2011) 057101. doi:10.1103/PhysRevE.63.057101.

[25] Y. Zhu, L. Hong, Z. Yang, W. Yong, Conservation-dissipation formalism of irreversible thermodynamics, J. Non-Equilib. Thermodyn. 40 (2015) 67-74. doi:10.1515/jnet-2014-0037. 
[26] S. R. de Groot, P. Mazur, on-equilibrium thermodynamics, North-Holland Publishing Company, Amsterdam, 1962.

[27] R. F. G. Visintin, R. Lapasin, E. Vignati, P. Antona, T. P. Lockhart, Rheological behavior and structural interpretation of waxy crude oil gels, Langmuir 21 (2005) 6240-6249. doi:10.1021/la050705k

[28] H. A. Barnes, The yield stress - a review or ' $\pi \alpha v \tau \alpha \rho \varepsilon \iota$ ' - everything flows?, J. Non-Newtonian Fluid Mech. 81 (1999) 133-178. doi:10.1016/ S0377-0257(98) 00094-9.

[29] H. B. Callen, Thermodynamics, Wiley, New York, 1960.

[30] W. Muschik, Internal variables in non-equilibrium thermodynamics, in: Recent Developments in Micromechanics, Springer-Verlag, Berlin Heidelberg, 1991, pp. 127-137. doi:doi:10.1007/978-3-642-84332-7_2.

[31] M. Grmela, D. Jou, J. Casas-Vázques, Non-linear and hamiltonian extended irreversible thermodynamics, J. Chem. Phys. 108 (1988) 7933-7945. doi:10.1063/1.476231. 